\title{
Ubiquitous User Assistance in a Tourist Information Server
}

\author{
Liliana Ardissono, Anna Goy, Giovanna Petrone, and Marino Segnan, \\ and Pietro Torasso ${ }^{\star}$ \\ Dip. Informatica, Università di Torino, Corso Svizzera 185, I-10149 Torino, Italy \\ \{liliana, goy, giovanna, marino, torasso\}@di.unito.it \\ http://www.di.unito.it/ seta $^{2}$
}

\begin{abstract}
This paper discusses the provision of ubiquitous services for desktop and handheld interfaces. The focus of the paper is on the interactive agenda offered by INTRIGUE, a tourist information server which assists the user in the organization of a tour by providing personalized recommendations of tourist attractions and helping the user to schedule her itinerary. The interactive agenda can be accessed both remotely, by interacting with the central server, and locally to the user's device.
\end{abstract}

\section{Background}

With the current expansion of wireless communications, several Web-based services have been extended to the mobile phone market, with special attention to contextawareness and to the adaptation of the user interface to the peculiarities of the various handset devices. However, only very basic, "real-time" services, such as the consultation of stock quotes, are accessed by mobile phone users, because wireless connections are extremely expensive. In contrast, the applications that can be executed locally to the device, such as music and games, are appreciated by a broader customer base. Although the situation might change in the future, at the current stage applications should rely on wireless network connections for the tasks that really need a remote connection [1].

In our recent research, we have addressed the design of system architectures for the development of services accessible both from desktop environments and handset devices, via remote connections to central servers, or local execution of tasks within the devices themselves. In these applications, persistent interaction contexts have to be managed to support the synchronization of the user's activities when switching from one device to the other [2]. We experimented our ideas with INTRIGUE (INteractive TouRist Information GUidE), a prototype information service presenting tourist information on Web browsers and WAP phones. This paper describes INTRIGUE's interactive agenda, which supports the tour scheduling by generating optimized itineraries on the basis of the user's temporal constraints, the attractions' opening times and their location. In this application, the persistent interaction context is represented by the user's itinerary, which can be consulted and modified during different sessions. The interactive agenda is downloadable and can run both in remote modality, by interacting with

\footnotetext{
* This paper has been published in LNCS n. 2347, Springer Verlag.
} 
局 is quite short. For yourself it is much eye-catching and it has high historical value.

医agenda palazzo Madama wowk For yourself it has high historical value.

IJagadd to

汿adagenda palazzo Carignano *k For children the visit is quite short. For yourself it has high historical value.

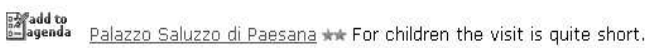

Fig. 1. Recommendation of attractions generated by INTRIGUE for a Web browser.

the tourist information server, and local modality, where the user can re-schedule her itinerary, without connecting to the server. This facility supports a quick revision of itineraries during the tour, therefore extending the role of our system, from tourist information service to interactive tourist guide, supporting the user during the visit of the attractions.

The paper is organized as follows: section 2 provides an overview of INTRIGUE. Section 3 and 4 describe the interactive agenda service and the ubiquitous access facilities offered by our system. Section 5 sketches the system architecture and the dynamic generation techniques exploited to manage the user interface. Finally, section 6 discusses some related work and concludes the paper.

\section{Overview of the System}

INTRIGUE provides multilingual information about tourist attractions and services, such as accommodation and food, in a geographical area. The main goal is assisting the user in the organization of a tour, by taking into account the possibly conflicting preferences of a group of people traveling together; for instance, a family with adults and children. Our current prototype presents information about the city of Torino and the surrounding Piedmont area, in Italian and in English [5]. The system supports the search for tourist attractions by offering orthogonal criteria, such as category-based and geographical search: e.g., the user can look for churches in Torino city, buildings in the area around Torino, and so forth. The search queries are created by exploiting a graphical interface hiding the details of the query specification language: the user browses a taxonomy of categories and selects the desired geographical area by clicking on a map, or by following suitable hypertextual links, depending on the device she is using.

Personalization strategies are applied to recommend the attractions best suiting the preferences of the tourist group. For instance, Figure 1 shows a recommendation list generated by our system: each item is a hypertextual link, which the user can follow 


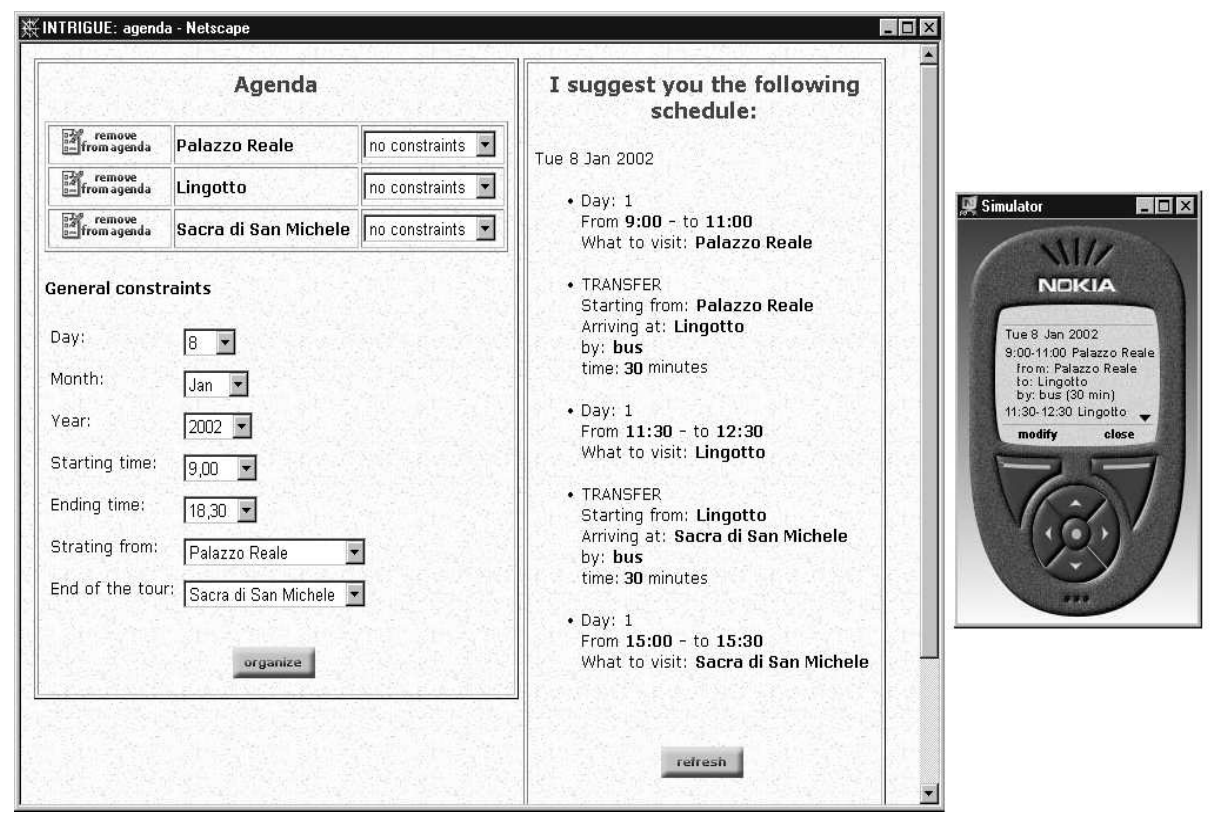

Fig. 2. Specifi cation of constraints and itinerary presentation in the interactive agenda: (a) desktop and (b) handheld interfaces.

to view a page presenting the most relevant information about the attraction. Moreover, each item is associated with a pictorial representation of its recommendation degree (stars) and, if relevant, with an explanation of the system's suggestion. Finally, an "add to agenda" button enables the user to include the item in the list of attractions to be scheduled in her itinerary.

\section{The Interactive Agenda}

The interactive agenda can be accessed by clicking a "View Agenda" button available in the recommendation pages. Given the set of attractions selected by the user, the agenda supports the specification of various types of constraints: e.g., day of visit, arrival/departure time and location, preferred time of visit. On the basis of the user's choices, the system tries to generate an itinerary that includes as many selected attractions as possible, complies with the attractions' opening times and the user preferences, and minimizes the overall transfer time. ${ }^{1}$ The presentation of an itinerary specifies, for

\footnotetext{
${ }^{1}$ The system applies constraint-solving techniques for the generation of candidate solutions, which are then compared for the selection of the one to be suggested on the basis of their total transfer times. The straight application of "shortest path" algorithms, such as the sales traveler one, is not possible, as they do not guarantee that the generated permutations of attractions satisfy temporal constraints like those concerning the opening time of attractions.
} 


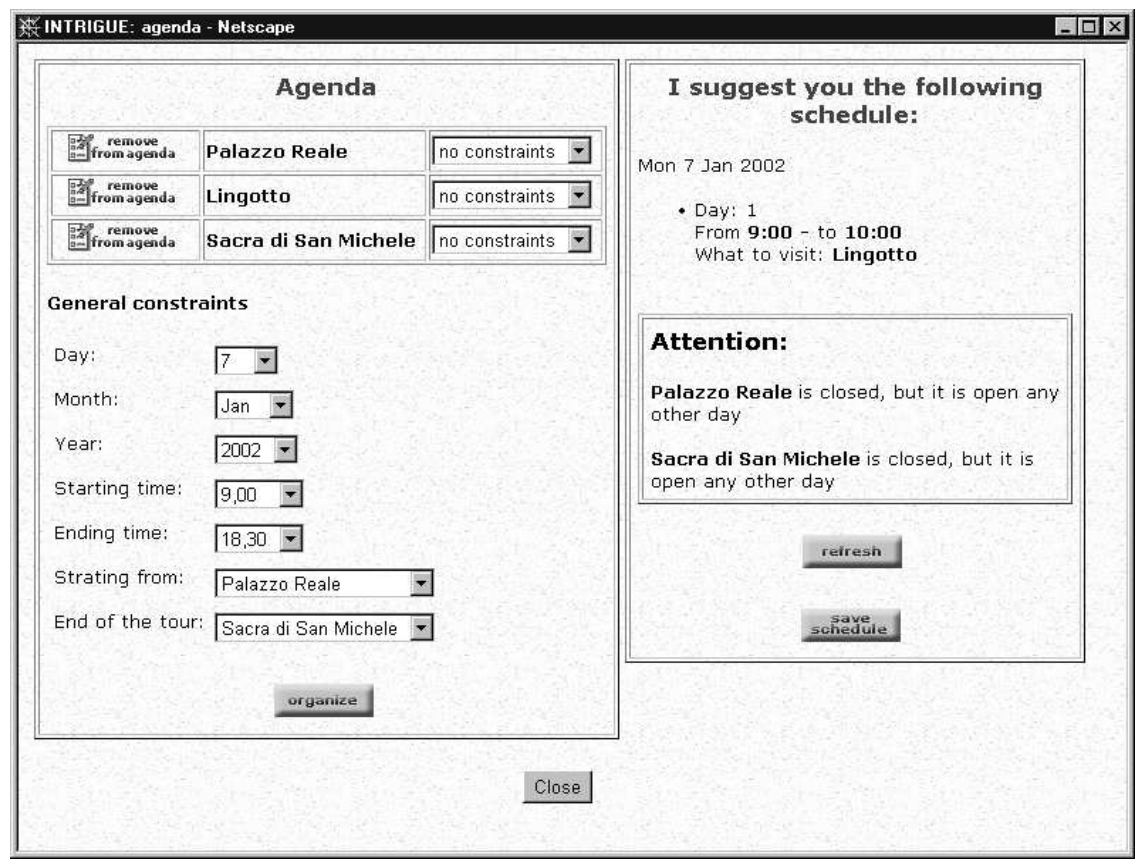

Fig. 3. Failure in the organization of an itinerary.

each attraction, the estimated transfer time to reach it, the time of the visit and its expected duration. Figures 2.(a) and 2.(b) present an itinerary for visiting Palazzo Reale, the Lingotto building and the Sacra di San Michele, in a situation where the user asked to start the visit from the Royal Palace and to end it at the Sacra. The presentation is different for desktop and handheld interfaces: in the former case, the system shows the user's constraints and the itinerary in the same page. In the latter, for space limitations, this information is compactly presented, in separate pages.

If the system fails in scheduling some of the selected attractions, it explains the problem and provides useful information to re-schedule the itinerary. For instance, the failure shown in Figure 3 is due to the fact that most tourist attractions are closed on Mondays. The user can solve the conflict by changing her selections. For example, she could try to visit other attractions, such as the parks, or she could modify her constraints, e.g., by changing the day of the tour. The interactive agenda enables the user to save an itinerary for further consideration ("save schedule" button in Figure 3).

\section{Ubiquitous Interaction with INTRIGUE}

As our system can be accessed by exploiting desktop computers and mobile phones, the user can use it during the organization of a tour and during the tour itself, in order to view the tourist catalog and generate itineraries for the visit of attractions. Moreover, the possibility to store the user's itinerary supports the any time revision of previously 


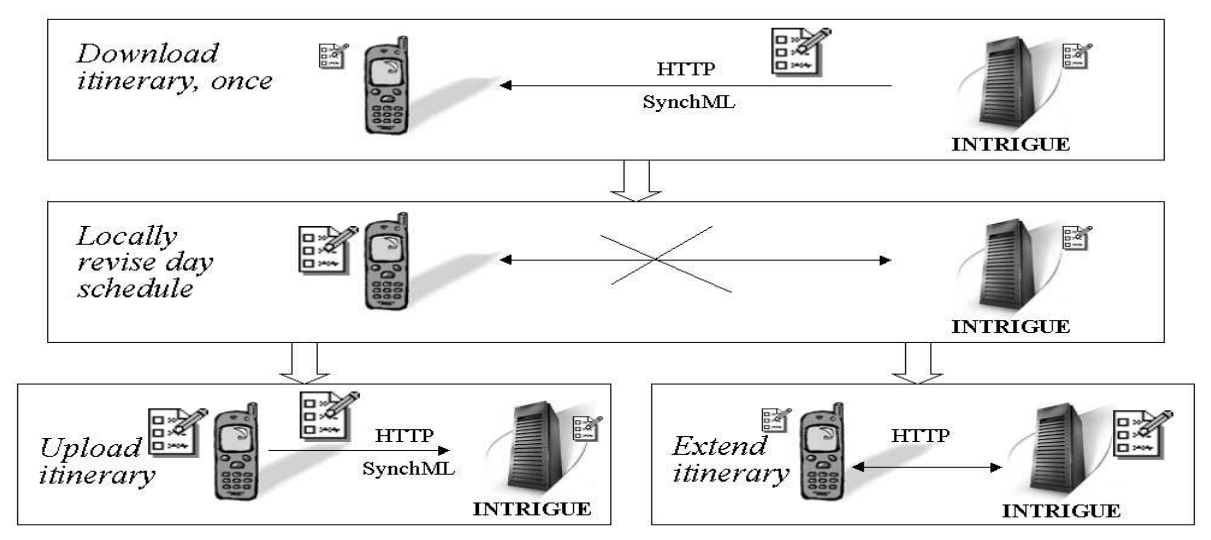

Fig. 4. Local interaction with INTRIGUE.

generated itineraries. However, the peculiarity of our system is the possibility to access some of its services locally to the user's device, which enables the access to the interactive agenda without a continuous connection to the central server. In this way, the user can consult her itinerary and possibly re-schedule it, by exploiting the mobile phone in a similar fashion to the way people use to run games on such devices.

The remote interaction with INTRIGUE is similar for desktop and handset devices: the user browses the catalog, selects tourist attractions to be visited and schedules itineraries. The central server stores the interaction context, which can include the user's itinerary, her last search query and maintains the information about the list of attractions satisfying the query (e.g., buildings around Torino, corresponding to the recommendation list shown in Figure 1). Moreover, the connection to the tourist information database supports the execution of further queries to retrieve new items that can be added to the agenda.

The local access to the services deserves further discussion. A possible scenario of a local use of the agenda is shown in Figure 4: the user connects to the remote server, downloads a previously generated itinerary on her handset and closes the interaction. Then, she opens the agenda to view and possibly reschedule her itinerary on the handset, in local modality. When the user later on connects again to the server, local changes to the itinerary are sent back to the networked data repository for permanent storage. ${ }^{2}$ In local access modality, the interactive agenda exploits its own information resources: the itinerary generation and scheduling capabilities are offered, but the user cannot add new items to the agenda, as only the information about the previous selections is available on the device. Therefore, if the user wants to consider new tourist attractions, she has to connect to the central server, by clicking a "modify $\rightarrow$ extend itinerary" link available in the interface of the handheld interactive agenda.

\footnotetext{
${ }^{2}$ In the fi gure, the currently used agenda is shown by a medium size icon; the possibly out of date one is shown as a small icon.
} 
The support of local and remote connections to the interactive agenda relies on the storage of an interaction context aimed at managing subsequent accesses to the system as portions of the same session.

- On the one hand, when saving an itinerary, the system also saves the information about the attractions selected by the user for further consultation. Moreover, the user's search query, representing the last focus of attention during the interaction, is saved. This information enables the user to connect to the central server for retrieving new items without browsing the catalog from the beginning. For instance, if the user initially searched for the buildings around Torino and wants to visit more places than those available in the agenda, she can directly access the recommendation page where the items are available; see Figure 1.

- On the other hand, when the user downloads an itinerary, the network connection is needed to download the itinerary and the information about the selected tourist attractions, which permanently reside in the database of the INTRIGUE server. After this synchronization phase, the agenda runs locally, without requiring further connections to the central server.

\section{Technical Aspects}

\subsection{Architecture}

INTRIGUE is based on the architecture described in [4], which has been extended to manage the interaction with mobile phones by exploiting the WAP protocol: we used the Nokia mobile phone simulator for our experiments. The generation of the devicedependent user interface relies on the exploitation of standard XML-based techniques; the local execution of the interactive agenda on handset devices is based on the exploitation of Java Micro Edition and of the synchronization facilities offered by such environment (SynchML [14]).

\subsection{Generation of the User Interface}

The User Interface (UI) of INTRIGUE can be viewed as a sequence of dynamically generated pages. The generation of each page is performed according to four distinct steps, listed in the following. While the first three steps are independent of the device the UI is implemented on, the last one generates the appropriate UI on the basis of the user's device.

- Selection of the information to be presented. Different information is selected for the various page types generated by the system. In a recommendation page (see Figure 1) the selected information consists of a list of attractions, sorted on the basis of the tourist group's preferences. In a page describing an attraction, such as the one in Figure 5, this information includes one or more sets of features describing the attraction from different points of view. Finally, the information selected for the agenda includes the list of attractions chosen by the user and the itinerary calculated by the system. 


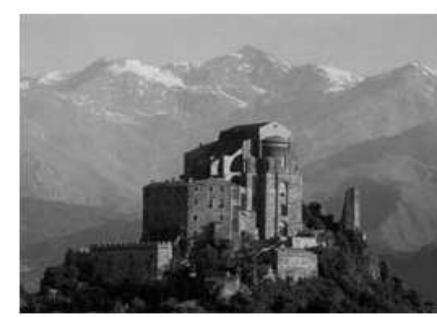

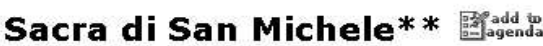

DESCRIPTION:

For Sacra di San Michele the visit is quite short, it has medium historical value, the documentation available is not present, it requires medium background knowledge, the access to visually impaired people is null, it has high artistic value, it has high natural value, there are no play areas available, it requires much seriousness, the architectural barriers are not easy to overcome and it is much eye-catching.

OPENING HOURS:

Open: Tue, Wed, Thu, Fri, Sat, Sun, 9:30-12:30; 15:00-17:00 (all the year)

INFORMATION:

- the guide is present

- average lenght of visit: 30 minutes

- phone: 011939130

- web address: www.sacradisanmichele.com/index.htm

- ticket price: Lit. 8000

- there are food and snacks available

- not present documentation

- the access to public is yes

- no credit cards accepted

- there are discount available

- there is no parking available

CHARACTERISTICS:

- type of monument: Churchs

- area: Torinese

- historical period: $400-1120$

- artistic current: Romanic

- town: Chiusa Di San Michele

Fig. 5. Portion of a page prefenting a tourist attraction for desktop interface.

- Generation of linguistic descriptions. The internal representation of the selected information is translated to natural language by exploiting efficient template-based NLG techniques supporting the production of simple sentences in Italian and English [3].

- Generation of the content structure of the page. A DTD defines the logical structure of the various types of page the system can produce; navigation pages, presentation pages, agenda pages, and so forth. An XML object (i.e., an XML instance document) representing the personalized content of the page, independent of the actual $\mathrm{UI}$, is produced.

- Surface generation. The UI is generated by transforming the XML object to a standard HTML, or WML page, depending on the user's device (desktop or mobile phone). In both cases, XSL stylesheets are exploited for the transformation. In contrast, when the user accesses the agenda in local modality, XSL stylesheets cannot be used, since the Java Micro Edition, running on mobiles, does not currently support standard tools for XSL processing such as Xalan. Taking also performance limitation of handset devices into account, we implemented a low-level WML generation module, based on light XML parsing (basically relying on SAX 1.0 APIs). 
In the transformation from XML to HTML/WML, the surface generator performs two choices:

- It selects the subset of content stored in the XML object to presented, given the space constraints of the display. For instance, the page describing the tourist attraction in Figure 5 is generated for a Web browser, where there are no serious space constraints: thus, all types of features are displayed. When generating the same type of page for a mobile, the surface generator omits the picture and selects only the most important types of features, leaving the rest available via "more info" links. For instance, both the "DESCRIPTION" paragraph (containing qualitative information about the item), and the "CHARACTERISTICS" section (reporting detailed information) are hidden, although they can be accessed via a link.

- It provides the layout information, deciding where and how, on the display, the various pieces of content should be placed.

\section{Related work and Discussion}

The dynamic, context-aware organization of a tour was initially proposed in the GUIDE system to support users in the real-time organization of their visit to the Lancaster town [12]. However, the architecture of such system is based on the exploitation of specialized hardware, physically located in the place to be visited, and on the use of special handset devices. In contrast, INTRIGUE is based on standard wireless connections and on the use of (Java-enabled) mobile phones. Moreover, the interactive agenda maintains persistent itineraries that can be consulted and updated by exploiting wired and wireless connections. Therefore, our system offers an effective ubiquitous service accessible from any place.

Ubiquitous computing has been interpreted by several researchers as a way to offer, on wireless connections, the same services already developed for internet-based browsers. As discussed in [8], the user interfaces offered by desktops, televisions and handset devices differ in size, interaction tools (e.g., keyboard, mouse, etc.) and usage environment (e.g., desktop, handheld device). In order to face this heterogeneity, the Adaptive Hypermedia community has developed adaptation techniques to tailor the interaction to the specific devices. For instance, interesting examples of services that the user can access both from desktop and WAP phone are provided in [17, 11,10,15]. Although these examples deeply differ from one another in the offered functionalities and underlying technologies, all of them exploit the user's device as a lightweight client, continuously interacting with a remote server, where the logic of the application resides. Moreover, they mainly interpret context-awareness as taking the user's physical (or temporal) coordinates into account.

Our proposal differs from these approaches in the following two main aspects.

- In addition to the basic service provision, which has to rely on a server-based management of the interaction with the user, we offer an interactive agenda and other services that the user can access locally to the device.

- We interpret context awareness and ubiquitous computing as a persistence property of the interaction with the user. In particular, the interactive agenda is modeled as a 
persistent object, that the user can create, update and carry with herself at any time, and along different interactions with the system, regardless the device she is using.

The first aspect is particularly relevant to the exploitation of handset devices, which are subject to important limitations: first, at the current stage of technological development, wireless connections are not reliable; therefore, long-lasting interactions, typically performed in the wired case, are problematic. Similarly, bandwidth is limited, so that there are clear demands for traffic minimization. Finally, in most countries wireless communications are very expensive; therefore, in order for users to appreciate the service, such connections should be limited to the services that cannot be offered off-line: e.g., the search for continuously updated information, such as stock quotations, and the management of business interactions, such as reservations and payment transactions. Although the first two issues might be overcome in few years, it is not clear whether the third one will be solved in the medium term. In order to develop successful applications, such constraints should be taken into serious account.

We have performed an initial testing of our system with users who helped us to improve its interface and functionalities. The users were happy about the system's explanation facilities, both regarding group recommendations and failures in itinerary generation, because they clarify the system's behavior, therefore increasing the trust in the system's recommendations. Moreover, users appreciated the possibility of saving itineraries and retrieving (and modifying) them in subsequent interactions, as this facility would enable them to use the interactive agenda during the tour. In contrast, there was some negative feedback in the organization of the device-dependent interaction with the user. For instance, the registration form for the specification of heterogeneous tourist groups, which was well-accepted during the desktop interaction, was considered too time consuming for a minibrowser. To address this issue, we have extended the system to support logging without registering: if the user logs in this way, she receives non personalized recommendations, but she can access the other facilities offered by the system, such as category and geographical search, itinerary scheduling and presentation of tourist attractions. Moreover, we will investigate the application of unobtrusive user modeling techniques aimed at the identification of the user's preferences on the basis of the observation of her browsing behavior $[6,9]$.

Our future work will include further testing of the system, in order to collect more usage information and hopefully to try the system with real mobile phones: the mobile functionalities of our system are currently tested by exploiting a the WAP simulator. Moreover, we will exploit further contextual information to enhance the itinerary generation capabilities; e.g., to take location and temporal information into account, as done in other systems $[7,16,2,13]$.

\section{References}

1. Special issue: The mobile internet. The Economist, (October 11, 2001) 27-54

2. G.D. Abowd and E.D. Mynatt. Charting past, present and future research in ubiquitous computing. ACM Transactions on Computer-Human Interaction, Special Issue on HCI in the new Millennium, 7(1) (2000) 29-58 
3. L. Ardissono and A. Goy. Dynamic generation of adaptive Web catalogs. In Lecture Notes in Computer Science, Vol. 1892: Adaptive Hypermedia and Adaptive Web-Based Systems. Springer-Verlag, Berlin (2000) 5-16

4. L. Ardissono, A. Goy, G. Petrone, and M. Segnan. A software architecture for dynamically generated adaptive Web stores. In Proc. 17th IJCAI, Seattle (2001) 1109-1114

5. L. Ardissono, A. Goy, G. Petrone, M. Segnan, and P. Torasso. Tailoring the recommendation of tourist information to heterogeneous user groups. In Third Workshop on Adaptive Hypertext and Hypermedia, Aarhus, Denmark (2001) 93-107

6. L. Ardissono and P. Torasso. Dynamic user modeling in a Web store shell. In Proc. 14th Conf. ECAI, Berlin (2000) 621-625

7. B.B. Bederson. Audio augmented reality: a prototype automated tour guide. In R. Mack J. Miller, I. Katz and L. Marks, editors, Conf. companion for the ACM Conf. on Human Factors in Computing Systems, Denver (1995) 210-211

8. E. Bergman, editor. Information Appliances and Beyond. Interaction design for consumer products. Morgan Kaufmann Publishers (1999)

9. D. Billsus and M. Pazzani. A personal news agent that talks, learns and explains. In Proc. 3rd Int. Conf. on Autonomous Agents, Seattle (1999) 268-275

10. D. Billsus, M. Pazzani, and J. Chen. A learning agent for wireless news access. In Proc. 2000 Int. Conf. on Intelligent User Interfaces, New Orleans (2000) 33-36

11. B. De Carolis, F. de Rosis, and S. Pizzutilo. Context-sensitive information presentation. In Proc. UM'2001 Workshop on User Modelling in Context-Aware Applications, Sonthofen, Germany (2001)

12. K. Cheverest, N. Davies, K. Mitchell, A. Friday, and C. Efstratiou. Developing a contextaware electronic tourist guide: some issues and experiences. In Proc. CHI'2000: Human factors in computing systems, The Hague, NL (2000) 17-24

13. A.K. Dey and D. Abowd. Towards a better understanding of context and context-awareness. In Proc. CHI2000 Workshop on the What, Who, Where, When and How of ContextAwareness, The Hague, NL (2000)

14. Y. Feng and J. Zhu. Wireless Java Programming with J2ME. SAMS (2001)

15. C. Paris, S. Wan, R. Wilkinson, and M. Wu. Generating personal travel guides - and who wants them? In Proc. 8th Int. Conf. on User Modeling Sonthofen, DE (2001) 251-253

16. D. Petrelli, A.De Angeli, and G. Convertino. A user centered approach to user modelling. In Proc. 7th Int. Conf. on User Modeling, Banff (1999) 255-264

17. D. Riecken, editor. Special Issue on Personalization, 43. Communications of the ACM (2000) 